\title{
Editorial: COVID-19: Mitigation Strategies and Their Implications for the Global Environment
}

\author{
Lei He ${ }^{1}$, Qunfang $\mathrm{Hu}^{2}$, Zhongmin $\mathrm{Liu}^{3}$ and Ying Zhang ${ }^{4 *}$ \\ ${ }^{1}$ College of Architecture and Urban Planning, Tongji University, Shanghai, China, ${ }^{2}$ Shanghai Institute of Disaster Prevention and \\ Relief, Tongji University, Shanghai, China, ${ }^{3}$ Shanghai East Hospital, School of Medicine, Tongji University, Shanghai, China, \\ ${ }^{4}$ College of Environment and Resource, Fuzhou University, Fuzhou, China
}

Keywords: COVID-19, mitigation strategy, environmental impact, community transmission, air pollutants

\section{Editorial on the Research Topic}

\section{COVID-19: Mitigation Strategies and Their Implications for the Global Environment}

\section{OPEN ACCESS}

Edited and reviewed by: Serena Zacchigna, International Centre for Genetic Engineering and Biotechnology, Italy

${ }^{*}$ Correspondence: Ying Zhang yzhang@fzu.edu.cn

Specialty section: This article was submitted to Environmental Economics and Management,

a section of the journal

Frontiers in Environmental Science

Received: 20 January 2022 Accepted: 04 February 2022

Published: 28 February 2022

Citation:

He L, Hu Q, Liu Z and Zhang Y (2022) Editorial: COVID-19: Mitigation Strategies and Their Implications for the Global Environment.

Front. Environ. Sci. 10:858607. doi: $10.3389 /$ fenvs.2022.858607
The COVID-19 pandemic is still wreaking havoc across the world. Actually, nobody could have foreseen that this horrible disaster could last more than 2 years and now nobody can predict when it will terminate. Some researchers suggested that SARS-CoV-2 might stay with us for a long time (Shaman and Galanti, 2020). To cope with this unprecedented global public health emergency, the academic community have devoted extensive efforts to understand the consequences and implications of COVID-19 on the global environment. This special issue was launched in July 2020, with the aim to highlight research achievements on the COVID-19 mitigation strategies and their long-term impact on the environment. The publications in this special issue mainly focus on the transmission of COVID-19 in communities, the interventions adopted by research institutions, the influence of environmental factors on the positive patients, and the impact of COVID-19 on aviation industry and air environment.

Hu et al. elucidate two transmission modes of COVID-19, i.e., the well-known direct person-toperson and the uncommon indirect media-to-person pathway. The latter one appears to be particularly relevant for the rapid spread of COVID-19 in communities with high population density. $\mathrm{Hu}$ et al. also propose three intervention approaches to interrupt the COVID-19 transmission in communities. Considering the mutation rate of SARS-CoV-2, this work is prospective and inspires further investigation in clustered communities with a poor sanitation.

An Iranian research group tried to apply a new intervention approach, named Intervention Map (IM), in dental schools in order to develop a comprehensive and structured mitigation strategy in research institutions. This work from Heidari et al. is expected to pioneer new global and national guidelines, aimed to cope with the future public health emergencies.

Based on the data of 434 COVID-19 positive patients in Al Kuwait Hospital, Dubai, UAE from January and June 2020, Hachim et al. correlated the temperature, wind speed, cloud cover, and precipitation rate with the clinical and laboratory parameter of COVID-19. They found that the weather-related variables played an important role on the dynamics of COVID-19 mortality and clinical outcome. For instance, hot weather could make infected people with comorbidities or elder patients more prone to develop aggressive inflammation and thus to experience serious complications or even death. In other words, the treatment and recovery plans of infected people should take weather-related factors into consideration.

During the outbreak of COVID-19 in China, Bao et al. analyzed the concentrations of three typical air pollutants (i.e., HC, CO, and NOx) from the LTO cycles for 22 airports in the Yangtze 
River Delta. Based on the temporal and spatial variation in the concentrations of these three pollutants, they found that the release of these three pollutants significantly decreased due to the COVID-19 pandemic, consistent with data from many other countries. Obviously, through the investigation of environmental response to COVID-19, the interplay between human and nature could be better understood and may trigger other potential research topics in the control of greenhouse gas.

The COVID-19 pandemic has brought major changes in both the human society and the global environment. Although we still do not know what we are going to face in the next month, it is important to summarize current experiences and lessons learnt by the measures so far implemented against COVID-19. We hope that the publications highlighted in this Editorial could provide a

\section{REFERENCES}

Shaman, J., and Galanti, M. (2020). Will SARS-CoV-2 Become Endemic? Science 370 (6516), 527-529. doi:10.1126/science.abe5960

Conflict of Interest: The authors declare that the research was conducted in the absence of any commercial or financial relationships that could be construed as a potential conflict of interest.

Publisher's Note: All claims expressed in this article are solely those of the authors and do not necessarily represent those of their affiliated organizations, or those of different perspective and new insight to better prevent and control the emerging infectious diseases in the future.

\section{AUTHOR CONTRIBUTIONS}

All authors listed have made a substantial, direct, and intellectual contribution to the work and approved it for publication.

\section{FUNDING}

This work was supported by the National Natural Science Foundation of China (51778437 and 51878510).

the publisher, the editors and the reviewers. Any product that may be evaluated in this article, or claim that may be made by its manufacturer, is not guaranteed or endorsed by the publisher.

Copyright $(2022 \mathrm{He}, \mathrm{Hu}$, Liu and Zhang. This is an open-access article distributed under the terms of the Creative Commons Attribution License (CC BY). The use, distribution or reproduction in other forums is permitted, provided the original author(s) and the copyright owner(s) are credited and that the original publication in this journal is cited, in accordance with accepted academic practice. No use, distribution or reproduction is permitted which does not comply with these terms. 